\title{
Characteristics of non-melanoma skin cancer depending upon occupational solar UV exposure dosage
}

\author{
Alexandra-Irina Butacu', Marc Wittlich², Swen Malte John ${ }^{3}$, Sabina Zurac ${ }^{4}$, \\ Horatiu Moldovan ${ }^{5}$, George-Sorin Tiplica ${ }^{6}$ \\ ${ }^{1}$ Dermatology Reseach Unit, Colentina Clinical Hospital, \\ "Carol Davila" University of Medicine and Pharmacy, Bucharest, Romania \\ ${ }^{2}$ Institute for Occupational Safety and Health of the German Social Accident Insurance, \\ Sankt Augustin, Germany \\ ${ }^{3}$ Department of Dermatology, Environmental Medicine and Health Theory, \\ University of Osnabrück, Osnabrück, Germany \\ ${ }^{4}$ Department of Pathology, Colentina Clinical Hospital, Bucharest, Romania \\ ${ }^{5}$ University of Medicine, Pharmacy, Science \& Technology of Targu-Mures, \\ Department of Occupational Medicine, Targu Mures, Romania \\ ${ }^{6} 2$ nd Department of Dermatology, Colentina Clinical Hospital, \\ "Carol Davila" University of Medicine and Pharmacy, Bucharest, Romania
}

\begin{abstract}
Objectives. Non-melanoma skin cancer (NMSC) is the most common human malignancy and is strongly related to solar ultraviolet (UV) radiation exposure. Occupational UV exposure is associated with a two-fold increased risk of developing NMSC, but still only few European countries recognize NMSC as an occupational disease. The study aims to analyze the clinical and histopathological features of NMSC with regard to personal cumulative occupational UV exposure doses.

Material and methods. A retrospective, analytical, comparative study was conducted during 2017-2019 with focus on outdoor workers presenting with NMSC. Occupational solar UV exposure dose was calculated based on the Wittlich algorithm and cumulative lifetime exposure of the general population of the same age was estimated according to German Standards of 130 SED/year. Subjects were divided in two groups (OW1 and OW2) according to differences between occupational exposure and estimated cumulative lifetime exposure of the general population. High-risk clinical and histopathological characteristics were compared between groups. Outcomes. The study included 25 consecutive outdoor workers diagnosed with BCC $(N=21)$ and SCC $(N=4)$. Occupational solar UV exposure doses were calculated for Romanian workers and identified mean values of 17695 SED two times higher than estimative leisure cumulative lifetime solar UV exposure for a person of the same age from the general population according to German standards of $130 \mathrm{SED} / y e a r$. Subjects were classified in two groups: group 1 (OW1) included subjects who were exposed to an additional occupational UV dose less than $50 \%$ of the cumulative lifetime exposure dose of a person of the same age from the general population according to German standards and group 2 (OW2) included subjects exposed to additional professional UV doses higher than 50\%. 12 subjects were included in OW 1 and 13 subjects in OW2.

Data analysis between groups identified significant statistical differences with regard to high-risk body locations and tumour size, present in $100 \%$ of OW2 and $58.3 \%$ of OW1 $(x 2(1, N=51)=6.771, p=0.015)$ and to high-risk histopathological subtypes, present in $56 \%$ OW2 and $33.3 \%$ OW1 $(x 2(1, N=51)=4.812, p=0.036)$. Ulceration was present on the histopathological report of all subjects in OW2. OW2 was associated with higher percentages of moderate to severe grades of actinic elastosis and of desmoplastic reaction and a more rapid evolution of lesions.

Conclusions: Outdoor workers in Romania are exposed to an occupational solar UV dose two times higher than the cumulative lifetime exposure dose of the general population of the same age which may receive up to $130 \mathrm{SED} / y e a r$ according to German standards and they bear an increased risk of NMSC. Occupational NMSC induced by UV exposure should be recognized as an occupational disease if the subject received more than $50 \%$ additional UV exposure at the workplace.
\end{abstract}

Keywords: skin cancer, UV occupational exposure, UV dosimetry

Corresponding author:

Assist. Prof. Alexandra-Irina Butacu, MD

E-mail: adabutacu@gmail.com
Article History:

Received: 10 March 2020

Accepted: 22 March 2020 


\section{INTRODUCTION}

Non-melanoma skin cancer (NMSC), also more precisely - referred to as keratinocyte cancer, is the most common human malignancy in both sexes and comprises basal cell carcinomas (BCC) and squamous cell carcinomas (SCC) [1].

Exposure to solar ultraviolet (UV) radiation is considered the most important risk factor in NMSC's etiopathogenesis [2] and is included since 2012 in the category of group 1 carcinogens (carcinogens to humans) by the World Health Organization [3]. Still, few European countries recognize occupationally UV-induced NMSC as an occupational disease, such as, for example, Germany, Denmark, and Italy. In 2016, only seven European countries recognized SCC as an occupational disease and only six recognized BCC, Romania not being part of any of these categories [4].

Furthermore, recent studies demonstrated a twofold increased risk of developing NMSC, including BCC and SCC for occupationally UV-exposed individuals when compared to the general population which only received leisure UV exposure [5,6].

The last decade provided new insights in occupationally-induced NMSC along with the introduction of UV-measuring personal dosimeters. This equipment permitted real quantitively UV measurements for outdoor professions and therefore an objective comparison between UV exposure in terms of occupation and geographical factors. For example, in Germany, the general population is exposed to $130 \mathrm{SED} /$ year (1 standard erythemal dose $=100 \mathrm{~J} / \mathrm{cm}^{2}$ ) while outdoor workers, for legal purposes, are considered to be exposed on average to an additional $300 \mathrm{SED} /$ year [7]. Taking these aspects into consideration, in Germany, NMSC is considered an occupational disease if an outdoor worker receives more than $40 \%$ additional UV exposure by his work to his lifetime UV exposure, since this is assumed to double the risk for NMSC development [8]. More precisely, lifetime UV exposure is calculated as years of first tumor detection by $130 \mathrm{SED} /$ year (for example, for first tumor detection at the age of 60 , the worker is expected to have received 7800 SED lifetime UV exposure). Furthermore, for every work year, occupational UV exposure dose is calculated as working years by $300 \mathrm{SED} /$ year (for example, for 15 outdoor working years, occupational exposure will be of 4500 SED). Therefore, in this example, the outdoor worker received $57 \%$ additional occupational UV exposure to his lifetime UV exposure and would be entitled to recognition of NMSC as an occupational disease (if the tumour occurred in occupationally UV exposed body locations).

In Romania, personal dosimetry studies were recently conducted and identified alarming UV doses for outdoor workers of up to $633 \mathrm{SED} /$ year [9], a value twice as high as the German values, but still this pathology is not recognized as an occupational disease. From the authors' knowledge, in Romania, no studies have correlated occupational UV doses with the presence and characteristics of NMSC, yet.

Therefore, this study aims to analyze the clinical and histopathological features of NMSC with regard to extrapolated personal cumulative occupational solar UV exposure doses.

\section{MATERIALS AND METHODS}

A retrospective, analytical, comparative study was conducted in a University Department of Dermato-venereology in Bucharest, with consecutive outdoor workers presenting with NMSC during 2017-2019.

A detailed personal history was obtained for all patients at the time of hospital admission and/or followed by additional telephone questions on a case to case basis. All patients were treated by surgical excision of tumors and all lesions underwent histopathological examinations.

Personal history was divided in two parts: (1) general information such as socio-demographic data, significant associated afflictions or risk factors and details about the evolution of lesions and (2) a thorough anamnesis based on work-related behavior which offered the possibility of determining occupational exposure dosage: hours per day and days per week spent at the work place, time intervals of outdoor activities, breaks, working years, geographical coordinates of the work place, applied sun protective measures and equipment. Occupational exposure dosage was calculated based on the Wittlich algorithm [8] - a German standardized instrument which calculates cumulative lifetime UV exposure and on recent Romanian studies [9], through which real-time measured data of UV exposure doses were obtained by personal dosimetry using the GENESIS UV system.

The Wittlich algorithm was applied for each subject included in the study. For Romania, recent studies determined $633 \mathrm{SED} /$ year [9] as mean exposure level which was used for calculating occupational solar UV exposure dosage as 633 SED times (*) number of working years. Furthermore, cumulative lifetime exposure of the general popu- 
lation of the same age according to German Standards was estimated as $130 \mathrm{SED} /$ year [7] times (*) number of years. All values are presented in SED. These data permitted a classification of subjects in two groups according to differences between occupational UV exposure dosage and cumulative lifetime exposure dose of the general population of the same age: group 1 (OW1) included subjects exposed to an additional occupational solar UV dose less than $50 \%$ of the cumulative lifetime exposure dose of a person of the same age from the general population according to German standards and group 2 (OW2) included subjects exposed to additional professional UV doses higher than 50\%.

Clinical data obtained from personal history and histopathological criteria from each patient's personal report were analyzed and correlated between groups OW1 and OW2. High-risk clinical and histopathological criteria were chosen based on National Comprehensive Cancer Centre (NCCN) recommendations [10,11]. High-risk clinical features included location and size (area L (trunk and extremities) $>20 \mathrm{~mm}$, area $\mathrm{M}$ (cheeks, forehead, scalp, neck, and pretibial region) $>10 \mathrm{~mm}$, area $\mathrm{H}$ (central face, eyelids, eyebrows, periorbital skin, nose, lips, chin, mandible, preauricular and postauricular skin/sulci, temple, ear, genitalia, hands, and feet) and high-risk histopathological criteria included high-risk subtypes (mixed infiltrative, basosquamous BCCs, adenoid SCC).

Other analysed clinical criteria included number of lesions, tumor diameter and evolution of lesions measured in months and other histopathological criteria included tumor thickness, ulceration, desmoplastic reaction and grades of associated actinic elastosis.

Data was analyzed using the software SPSS for Windows version 20 (SPSS, Chicago, IL, USA). Mean values and standard deviations (SD) were calculated for numerical data. Statistical significance was tested for categorical data by Chi-square analysis $\left(\chi^{2}\right)$, corrected by Fisher' exact method in selected cases. Mean values of numerical data were compared using Independent-samples T test (Levene's test for Equality of Variances). $\mathrm{P}<0.05$ was considered to indicate statistical significance.

\section{OUTCOMES}

The study included 25 outdoor workers of various occupations such as construction and agriculture workers, outdoor sports teachers, workers of the oil industry and outdoor military personnel. Males were predominant (18 males, 69.2\%) with only 7 females (26.7\%). 15 (57.7\%) subjects came from urban environments and 10 (38.5\%) from rural environments. Mean age of outdoor workers was 68.92 (SD 12.09, $\min 35$, $\max 93$ ). Studied lesions included $21(80.8 \%)$ BCC and $4(15.4 \%)$ SCC.

Occupational UV exposure doses identified mean values of 17,695 SED (SD 6,449, $\min 3,165$, $\max 25,320)$, two times higher than mean values of 8,959 SED (SD 1572, min 4550, $\max 12090$ ) for estimated leisure cumulative lifetime UV exposure for a person of the same age from the general population according to German standards. A detailed description of identified and estimated doses is presented in Figure 1. Obtained solar UV data permitted identification of 12 subjects (48\%) in group OW1 and 13 subjects (52\%) in group OW 2.

Analysis of high-risk clinical and histopathological criteria (Table 1) between groups identified significant statistical differences with regard to high-risk locations and size, present in $100 \%$ of OW2 and $58.3 \%$ of OW $1(\chi 2(1, \mathrm{~N}=51)=6.771, \mathrm{p}$ $=0.015)$ and to high-risk histopathological subtypes, present in 56\% OW2 and $33.3 \%$ OW 1 , respectively $(\chi 2(1, \mathrm{~N}=51)=4.812, \mathrm{p}=0.036)$.

In OW $1,33.3 \%$ subjects associated low-risk clinical criteria such as location and size. Most frequent high-risk location and size was area $M>10$ $\mathrm{mm}$ in both groups: $33.3 \%$ of subjects for OW 1 and $69.2 \%$ for OW2 (Table 2). Low-risk histopathological subtypes were more frequently identified in OW1 as nodular BCC in 50\% of cases; in OW2, most common high-risk histopathological subtype was mixed infiltrative BCC (61.5\%) (Table 3).

Table 4 presents significant statistical differences between groups in terms of presence of ulceration and presence of moderate to severe actinic elastosis and desmoplastic reaction. Ulceration was present on the histopathological report of all subjects in OW2 $\left(\chi^{2}(1, \mathrm{~N}=51)=12.745, \mathrm{p}<0.001\right)$. Moderate to severe grades of actinic elastosis were identified in $92.3 \%$ subjects from OW2 and in $75 \%$ of subjects from OW1 $(\chi 2(1, \mathrm{~N}=51)=7.164$, $\mathrm{p}=0.017)$. Moderate to severe grades of desmoplastic reaction were present in $84.6 \%$ lesions from OW2 and in 58.3\% lesions from OW1 ( $\chi^{2}(1$, $\mathrm{N}=51)=4.043, \mathrm{p}=0.045$ ).

Table 5 identified significant statistical differences regarding diameter, tumor thickness and evolution. OW2 associated higher mean diameters of lesions ( 7.26 compared to $4.47 ; \mathrm{t}_{\text {obt }}(23)=-2.49, \mathrm{p}$ $=0.20)$, increased mean values of tumor thickness on the histopathological report (1.92 compared to 
- Occupational cumulative exposure (SED) = Estimative lifetime cumulative exposure (SED)

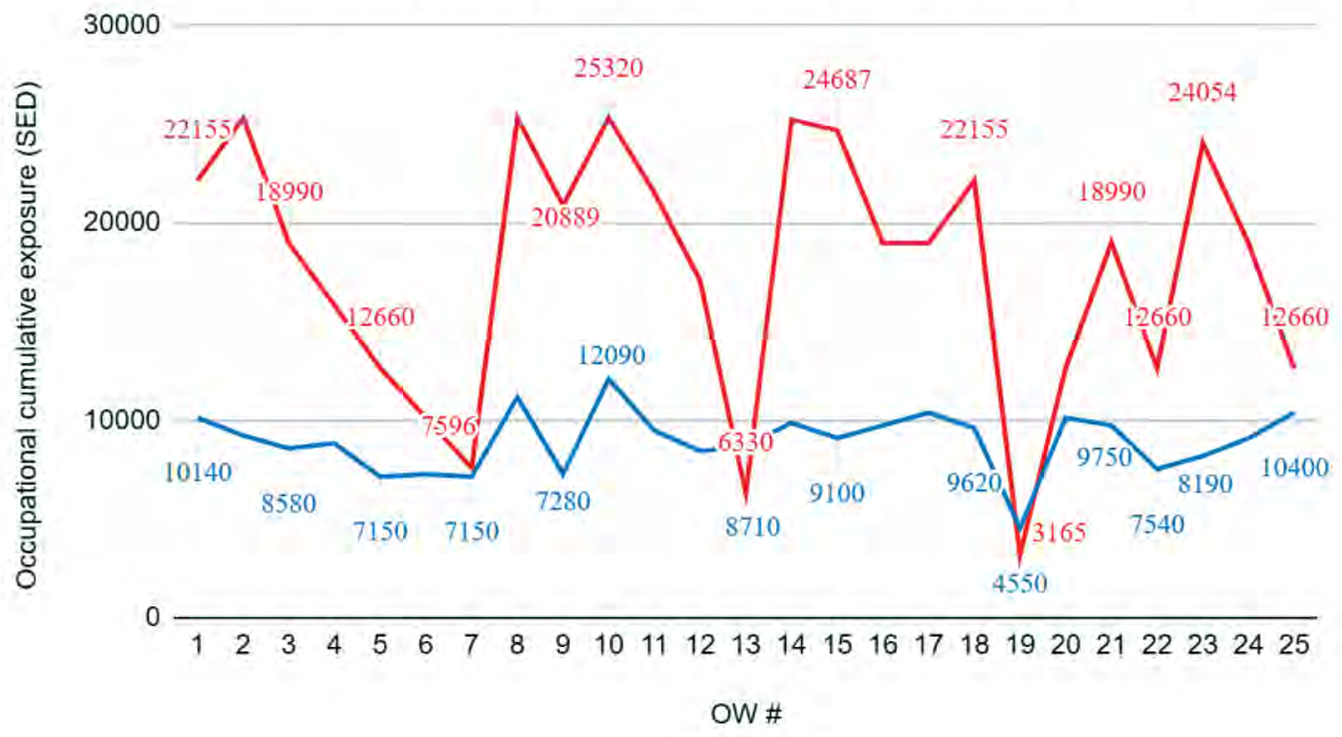

FIGURE 1. Differences between occupational cumulative UV exposure doses of subjects and lifetime cumulative UV exposure doses for the general population of the same age

TABLE 1. Overview of high-risk clinical and histopathological characteristics

\begin{tabular}{|l|c|c|c|c|}
\hline High-risk Characteristics & $\mathbf{N}$ & $\%$ & $\chi^{2}(\mathbf{1}, \mathbf{N}=\mathbf{2 5})$ \\
\hline \multirow{2}{*}{ High-risk location and size* } & OW1 & 7 & $58.3 \%$ & \multirow{2}{*}{$6.771(\mathbf{p}=\mathbf{0 . 0 1 5})$} \\
\cline { 2 - 4 } & OW2 & 13 & $100 \%$ & \\
\hline \multirow{2}{*}{ High-risk histological subtype* } & OW1 & 4 & $33.3 \%$ & \multirow{2}{*}{$4.812(\mathbf{p}=\mathbf{0 . 0 3 6})$} \\
\cline { 2 - 4 } & OW2 & 10 & $56 \%$ & \\
\hline
\end{tabular}

*According to NCCN

TABLE 2. Difference between high-risk NMSC clinical criteria between groups of outdoor workers

\begin{tabular}{|l|c|c|c|c|c|}
\hline \multirow{2}{*}{} & \multicolumn{5}{|c|}{ Clinical criteria: High-risk location and size* } \\
\cline { 2 - 6 } & Low-risk & Area L>20 $\mathbf{~ m m}$ & Area $\mathbf{M}>\mathbf{1 0} \mathbf{~ m m}$ & Area H & Total \\
\hline OW1 & $4(33.3 \%)$ & $2(16.7 \%)$ & $4(33.3 \%)$ & $2(16.7 \%)$ & 12 \\
\hline OW2 & 0 & 0 & $9(69.2 \%)$ & $4(30.8 \%)$ & 13 \\
\hline
\end{tabular}

*According to NCCN

TABLE 3. Difference between high-risk NMSC histopathological subtypes between groups

\begin{tabular}{|l|c|c|c|c|c|c|}
\hline \multirow{2}{*}{} & \multicolumn{6}{|c|}{ Histopathological criteria: Subtypes* } \\
\cline { 2 - 7 } & Nodular BCC & Superficial BCC & Infiltrative mixed BCC & Metatypical BCC & Well-dif. SCC & Poorly dif. SCC \\
\hline OW1 & $6(50 \%)$ & $1(8.3 \%)$ & $2(16.7 \%)$ & $1(8.3 \%)$ & 1 & 1 \\
\hline OW2 & $2(15.4 \%)$ & 0 & $8(61.5 \%)$ & $1(7.7 \%)$ & 1 & 1 \\
\hline
\end{tabular}

*According to NCCN

TABLE 4. Differences between additional high-risk NMSC histopathological criteria

\begin{tabular}{|l|c|c|c|}
\hline Histopathological high-risk criteria & OW1 N (\%) & OW2 N (\%) & $\boldsymbol{\chi}^{\mathbf{2}}(\mathbf{1}, \mathbf{N}=\mathbf{2 5})$ \\
\hline Presence of ulceration & $4(33.3 \%)$ & $13(100 \%)$ & $\mathbf{1 2 . 7 4 5}(\mathbf{p}<\mathbf{0 . 0 0 1})$ \\
\hline Actinic elastosis (moderate-severe) & $12(75 \%)$ & $21(92.3 \%)$ & $\mathbf{7 . 1 6 4}(\mathbf{p}=\mathbf{0 . 0 1 7})$ \\
\hline Desmoplastic reaction (moderate-severe) & $7(58.3 \%)$ & $11(84.6 \%)$ & $\mathbf{4 . 0 4 3}(\mathbf{p}=\mathbf{0 . 0 4 5})$ \\
\hline
\end{tabular}


TABLE 5. Differences between additional characteristics between groups of outdoor workers (OW)

\begin{tabular}{|c|c|c|c|c|c|}
\hline Characteristic & Groups & $\mathbf{N}$ & Mean & SD & $t_{o b t}(23)$ \\
\hline \multirow{2}{*}{ Diameter (mm) } & ow1 & 12 & 10.75 & 4.47 & \multirow{2}{*}{$-2.49(p=0.020)$} \\
\hline & OW2 & 13 & 16.85 & 7.26 & \\
\hline \multirow{2}{*}{ Tumor thickness (mm) } & OW1 & 12 & 1.57 & 0.98 & \multirow{2}{*}{$-2.08(p=0.048)$} \\
\hline & OW2 & 13 & 2.86 & 1.92 & \\
\hline \multirow{2}{*}{ Evolution (months) } & OW1 & 12 & 18.75 & 8.30 & \multirow{2}{*}{$4.74(p<0.001)$} \\
\hline & OW2 & 13 & 7.15 & 2.881 & \\
\hline \multirow{2}{*}{ Number of lesions } & OW1 & 12 & 1.33 & 0.65 & \multirow{2}{*}{$-1.01(p=0.319)$} \\
\hline & OW2 & 13 & 1.85 & 1.62 & \\
\hline
\end{tabular}

$\left.0.98 ; \mathrm{t}_{\text {obt }}(23)=-2.08, \mathrm{p}=0.48\right)$ and a more rapid evolution (mean value of 18.75 months in OW1 and 7.15 months in $\left.\mathrm{OW} 2 ; \mathrm{t}_{\text {obt }}(23)=4.74, \mathrm{p}<0.001\right)$. Number of lesions was not statistically different between groups $\left(\mathrm{t}_{\mathrm{obt}}(23)=-1.01, \mathrm{p}=0.319\right)$.

\section{DISCUSSION}

The impact of occupational solar UV radiation exposure is a topic of great interest worldwide with recent literature studies emphasizing the long-term health consequences of cumulative UV exposure on both skin, with the induction of NMSC and melanomas and on other organs such as the eyes, increasing the risk of cataract and age-related macular degeneration [12].

Furthermore, the constant depletion of the atmospheric ozone layer in the last few decades caused values of UV measurements at sea level up to 15 times higher when compared to satellite estimations, significantly increasing the risk of skin cancer pathology and associated long-term associated morbidity and mortality [13].

The risk of developing NMSC may also be directly linked to types of profession. Epidemiological studies presented a stratification of NMSC's risk according to occupation. The highest risk of developing NMSC was associated with mountain guides, followed by agriculture workers and gardeners [14].

Studies with focus on personal dosimetry permitted the identification of real-time quantitively data on European outdoor workers with alarming results. For example, outdoor workers in Denmark are exposed to $5 \mathrm{SED} /$ day [15], in Switzerland up to 28.6 SED/day [16], in Spain 6.11 SED/day [17], in France 1.19 SED/day [18] and in Romania from 6.4 SED/day [19] up to $19 \mathrm{SED/day} \mathrm{[20].} \mathrm{These}$ data show high variability between European countries and suggest the necessity of stratifying NMSC according to exposure dosage in order to obtain prediction models of NMSC's characteristics with regards to occupation and occupational protective behavior. Furthermore, these aspects will offer the possibility of developing specific sun protective measures for each occupation.

Recognizing NMSC as an occupational disease across Europe is a first essential step in preventing this affliction. Currently, up to $90 \%$ of the allocated healthcare budget for NMSC is invested in treatment and only $10 \%$ in prevention [21]. Therefore, development of NMSC is associated with workers absenteeism, reduced productivity at the workplace and even job loss; however, preventive measures are available and can be efficiently applied [22]. The lack of sun smart protective behavior at the work place is observed throughout Europe, with most outdoor workers using photoprotection during holidays, but not during working hours [23], noticing a big gap in the culture of prevention.

Characterizing occupationally-induced NMSC has been previously attempted, identifying the "mask area" of the face as the most common tumor localization and mixed with an aggressive component as the most frequent histopathological subtype [24].

This study related NMSCs clinical and histopathological characteristics in outdoor workers to differences between their occupational solar UV exposure compared to the cumulative lifetime exposure of the general population of the same age according to German standards of $130 \mathrm{SED} /$ year. This is the first such attempt in Romania to the best of the authors' knowledge. The subjects were categorized in two groups similarly with the German NMSC recognition system, in which NMSC is considered occupationally-induced if the subject received more than $40 \%$ additional UV exposure by his work compared to his lifetime UV exposure. Since leisure solar UV dosage in Romania is not available, lifetime exposure was calculated based on the German standards of $130 \mathrm{SED} /$ year. Addi- 
tionally, taken into consideration that leisure UV exposure in Romania may be higher than Germany since the occupational UV exposure is higher, the threshold for categorizing subjects was set at 50\% additional UV exposure at the workplace to the cumulative lifetime UV exposure.

In Romania, outdoor workers exposed to $50 \%$ additional occupational solar UV doses to the cumulative lifetime UV exposure showed statistically significant higher percentages of high-risk clinical and histopathological criteria. These included localization on high-risk areas and higher dimensions of tumors, higher percentages of high-risk histopathological subtypes, more frequent association with ulceration, high grades of desmoplastic reaction and actinic elastosis as well as a more rapid evolution of lesions.

The authors propose this classification system in Romania for recognizing NMSC, with $633 \mathrm{SED} /$ year as reference value for occupational UV exposure dose and with recognition of NMSC as an occupational disease as long as the subject receives more than $50 \%$ additional UV exposure at the workplace to his lifetime cumulative exposure dose according to German standards of $130 \mathrm{SED} /$ year.

Last but not least, the authors draw attention to the necessity of introducing NMSC in National Cancer Registries, as well as in the national list of occupational diseases, two topics of great interest

\section{REFERENCES}

1. Small J, Barton V, Peterson B, Alberg AJ. Keratinocyte Carcinoma as a Marker of a High Cancer-Risk Phenotype. Advances in Cancer Research, vol. 130, Academic Press Inc.; 2016, p. 257-91.

2. Saladi RN, Persaud AN. The causes of skin cancer: A comprehensive review. Drugs of Today 2005;41:37-53.

3. IARC Working Group on the Evaluation of Carcinogenic Risks to Humans., International Agency for Research on Cancer. A review of human carcinogens. Radiation. International Agency for Research on Cancer; 2012. Available at: https://monographs.iarc.fr/wp-content/ uploads/2018/06/mono90.pdf. [Accessed on 14 February 2020].

4. Ulrich C, Salavastru C, Agner T, Bauer A, Brans R, Crepy MN et al. The European Status Quo in legal recognition and patient-care services of occupational skin cancer. Journal of the European Academy of Dermatology and Venereology 2016;30:46-51.

5. Schmitt J, Haufe E, Trautmann F, Schulze HJ, Elsner P, Drexler H, et al. Is ultraviolet exposure acquired at work the most important risk factor for cutaneous squamous cell carcinoma? Results of the population-based case-control study FB-181. British Journal of Dermatology 2018;178:462-72.

6. Schmitt J, Haufe E, Trautmann F, Schulze HJ, Elsner P, Drexler H et al. Occupational UV-exposure is a major risk factor for basal cell carcinoma: Results of the population-based case-control study FB-181. Journal of Occupational and Environmental Medicine 2018;60:36-43.

7. Diepgen TL, Brandenburg S, Aberer W, Bauer A, Drexler H, Fartasch $M$ et al. Skin cancer induced by natural UV-radiation as an occupational disease - requirements for its notification and recognition. not only locally but also at an international level [25].

\section{CONCLUSIONS}

1. Outdoor workers in Romania are exposed to an occupational solar UV dose two times higher than the cumulative lifetime exposure dose of the general population of the same age which may receive up to $130 \mathrm{SED} /$ year according to German standards.

2. Outdoor workers in Romania exposed to higher occupational solar UV doses bear an increased risk of NMSC with high-risk location and sizes, high-risk histopathological subtypes, presence of ulceration and high grades of actinic elastosis and desmoplastic reaction.

3. Outdoor workers exposed to higher occupational UV doses are associated with higher lesion diameters, increased tumor thickness and a more rapid evolution of lesions.

4. Occupational NMSC induced by UV exposure should be recognized as an occupational disease if the subject received more than $50 \%$ additional UV exposure at the workplace compared to his lifetime cumulative UV exposure dose of 130 $\mathrm{SED} /$ year, calculated on the base of $633 \mathrm{SED} /$ year as a mean exposure dose for occupational solar UV exposure in Romanian workers.

Conflict of interest: none declared Financial support: none declared

Journal Der Deutschen Dermatologischen Gesellschaft $=$ Journal of the German Society of Dermatology: JDDG 2014;12:1102-6.

8. Wittlich M, Westerhausen S, Kleinespel P, Rifer G, Stöppelmann W. An approximation of occupational lifetime UVR exposure: Algorithm for retrospective assessment and current measurements. Journal of the European Academy of Dermatology and Venereology 2016; 30:27-33.

9. Wittlich M, John S, Tiplica G, Sălăvăstru C, Butacu A, Modenese A, et al. Personal solar ultraviolet radiation dosimetry in an occupational setting across Europe. Journal of the European Academy of Dermatology and Venereology 2020.

10. National Comprehensive Cancer Center. NCCN clinical practice guidelines in oncology; basal cell carcinoma (V1.2017). Available at: www.ncen.org.

11. National Comprehensive Cancer Center. NCCN clinical practice guidelines in oncology; squamous cell carcinoma (V1.2017). Available at: www.nccn.org.

12. Modenese A, Korpinen L, Gobba F. Solar radiation exposure and outdoor work: An underestimated occupational risk. International Journal of Environmental Research and Public Health 2018;15.

13. Little MP, Tatalovich Z, Linet MS, Fang M, Kendall GM, Kimlin MG. Improving Assessment of Lifetime Solar Ultraviolet Radiation Exposure in Epidemiologic Studies: Comparison of Ultraviolet Exposure Assessment Methods in a Nationwide U.S. Occupational Cohort. Photochemistry and Photobiology 2018;94:1297-307.

14. Zink A, Tizek L, Schielein M, Böhner A, Biedermann T, Wildner M. Different outdoor professions have different risks - a cross-sectional study comparing non-melanoma skin cancer risk among farmers, 
gardeners and mountain guides. Journal of the European Academy of Dermatology and Venereology 2018; 32:1695-701.

15. Grandahl K, Mortensen OS, Sherman DZ, Køster B, Lund PA, Ibler $\mathrm{KS}$ et al. Solar UV exposure among outdoor workers in Denmark measured with personal UV-B dosimeters: Technical and practical feasibility. BioMedical Engineering Online 2017;16.

16. Antoine M, Pierre-Edouard S, Jean-Luc B, David V. Effective exposure to solar UV in building workers: Influence of local and individual factors. Journal of Exposure Science and Environmental Epidemiology 2007; 17:58-68.

17. Serrano MA, Cañada J, Moreno JC. Solar UV exposure in construction workers in Valencia, Spain. Journal of Exposure Science and Environmental Epidemiology 2013; 23:525-30.

18. Boniol M, Koechlin A, Boniol M, Valentini F, Chignol MC, Doré JF, et al. Occupational UV exposure in French outdoor workers. Journal of Occupational and Environmental Medicine 2015; 57:315-20.

19. Moldovan HR, Wittlich M, John SM, Brans R, Tiplica GS, Salavastru C, Voidazan ST, Duca RC, Fugulyan E, Horvath G, Alexa A, Butacu Al. Exposure to solar UV radiation in outdoor construction workers using personal dosimetry. Environmental Research. 2019 Nov 27:108967.

20. Lungu Al, Butacu Al, Manole I, Suru A, Tiplica GS, Salavastru CM. Dosimeters: measuring occupational UV exposure's involvement in skin cancer development. Romanian Journal of Military Medicine. 2017; Vol. CXX, No.1.

21. Gordon LG, Rowell D. Health system costs of skin cancer and cost-effectiveness of skin cancer prevention and screening: A systematic review. European Journal of Cancer Prevention 2015; 24:141-9.

22. Mofidi A, Tompa E, Spencer J, Kalcevich C, Peters CE, Kim J et al. The economic burden of occupational non-melanoma skin cancer due to solar radiation. Journal of Occupational and Environmental Hygiene 2018; 15:481-91.

23. Grandahl K, Ibler KS, Laier GH, Mortensen OS. Skin cancer risk perception and sun protection behavior at work, at leisure, and on sun holidays: A survey for Danish outdoor and indoor workers 11 Medical and Health Sciences 1117 Public Health and Health Services. Environmental Health and Preventive Medicine 2018;23.

24. Salavastru CM, Ulrich C, Cretu S, Moldovan HR, Tiplica GS. The experience of a tertiary referral centre in Romania on basal cell carcinomas in outdoor workers: why to assess? Journal of the European Academy of Dermatology and Venereology. 2016 Apr; 30:12-6.

25. Zanetti R, Sacchetto L, Coebergh JW, Rosso S. To accelerate cancer prevention in Europe: Challenges for cancer registries. European Journal of Cancer 2018; 104:151-9. 